\title{
Course for undergraduate students: analysis of the retinal image quality of a human eye model
}

\section{Maria del Mar Pérez, Ana Yebra, Alicia Fernández- Oliveras, Razvan Ghinea, Ana lonescu, et al.}

Maria del Mar Pérez, Ana Yebra, Alicia Fernández-Oliveras, Razvan Ghinea, Ana M lonescu, Juan C. Cardona, "Course for undergraduate students: analysis of the retinal image quality of a human eye model," Proc. SPIE 9289, 12th Education and Training in Optics and Photonics Conference, 92890R (17 July 2014); doi: 10.1117/12.2070769

Event: 12th Education and Training in Optics and Photonics Conference, 2013, Porto, Portugal 


\title{
Course for undergraduate students: analysis of the retinal image quality of a human eye model
}

\author{
María del Mar Pérez*, Ana Yebra, Alicia Fernández-Oliveras, Razvan Ghinea, Ana M. Ionescu, \\ Juan C. Cardona \\ Departamento de Óptica, Facultad de Ciencias, Universidad de Granada. Edificio Mecenas, Campus \\ Fuentenueva s/n. 18071 Granada (SPAIN)
}

\begin{abstract}
In teaching of Vision Physics or Physiological Optics, the knowledge and analysis of the aberration that the human eye presents are of great interest, since this information allows a proper evaluation of the quality of the retinal image. The objective of the present work is that the students acquire the required competencies which will allow them to evaluate the optical quality of the human visual system for emmetropic and ammetropic eye, both with and without the optical compensation. For this purpose, an optical system corresponding to the Navarro-Escudero eye model, which allows calculating and evaluating the aberration of this eye model in different ammetropic conditions, was developed employing the OSLO LT software. The optical quality of the visual system will be assessed through determinations of the third and fifth order aberration coefficients, the impact diagram, wavefront analysis, calculation of the Point Spread Function and the Modulation Transfer Function for ammetropic individuals, with myopia or hyperopia, both with or without the optical compensation. This course is expected to be of great interest for student of Optics and Optometry Sciences, last courses of Physics or medical sciences related with human vision.
\end{abstract}

Keywords: Optics, Retinal Image Quality, biomedical optics, inter-disciplinary courses

\section{THEORETICAL BACKGROUND}

During the past years there is an increasing interest in evaluating the optical quality and optical aberrations of the eye. It is well known that the optical quality of the eye is limited by its optical aberrations, diffraction and scatterring ${ }^{1}$. A large part of the defects found in the retinal image have been studied and properly corrected during the past years, but only recently it became possible to accurately measure the aberrations of the eye. This, complemented with the constant development and improvement of refractive surgery, it might be possible to provide, in the near future, the adequate tools to correct even certain types of irregular astigmatism.

Aberration theory has been widely studied in rotationally symmetric systems, for optical design and also for testing purposes ${ }^{2,3}$. In conventional optical systems, the analytical expression of the aberration can be obtained as a function of several parameters, such as radius, asphericity, thickness of surface and refractive index of the media ${ }^{2,4}$. However, since the ocular optical system is formed by complex and non-symmetrical surfaces, the wave aberration of the optical system of the eye is typically obtained by means of a discrete sampling and measurement of a set of local aberrations, which are then fit to a functional expansion of the wave aberration. The wave aberration is in general a complex surface function, and it is usually described as a polynomial expansion. In this sense, the Zernike polynomial expansion, first introduced by Frits Zernike in interferometric contrastphase microscopy ${ }^{5}$, has become the standard to represent ocular wave aberration data. The main advantages of the set of Zernike polynomials is that they are orthonormal in a unit circle, and that they can be easily related to magnitudes typically used in optometry, such as defocus or astigmatism. The Zernike coefficients represent the contributions of each specific term to the wave aberration. The Optical Society of America has established a set of recommendations ${ }^{6}$, in terms of order, sign and normalization when reporting the Zernike polynomials.

*mmperez@ugr.es; phone (34) 9582416164; fax (34) 958248533

12th Education and Training in Optics and Photonics Conference, edited by

Manuel F. P. C. Martins Costa, Mourad Zghal, Proc. of SPIE Vol. 9289, 92890R

(c) 2014 SPIE, OSA, IEEE, ICO · doi: 10.1117/12.2070769

Proc. of SPIE Vol. 9289 92890R-1 
Not all the optical aberrations are visually significant. For example, zero-order aberration, such as the piston, is a constant added to the global wavefront and does not affect the overall optical quality of the system while first-order aberrations, such as tilt, produces a global shift of the retinal image which is canceled in foveal vision. However, it is well known that the quality of the retinal image is mainly influenced by the second-order aberrations, also called refractive errors. These spherocylindrical focus errors include myopia, hyperopia, and regular astigmatism and all of them can be compensated or corrected with prescription glasses, contact lenses or refractive surgery.

Higher order aberrations also affect the image quality, although to a smaller extent (about 10\% of the eye's total aberrations). Among the numerous higher-order aberrations, only the spherical aberration, coma and trefoil present certain clinical relevance. Measurements performed in normal eyes have proven that the lens has a compensatory effect on the spherical aberration of the cornea ${ }^{7,8}$. Also, compensation for aberrations between the cornea and intraocular optics in cases of astigmatism and horizontal coma ${ }^{7}$ have been reported. Recently, it has been determined that there is a strong correlation between the wavefront aberration pattern and the accommodative response of the eye ${ }^{9,10}$. In this sense, unaccomodated eyes present positive spherical aberration while accommodated eyes present a trend toward negative spherical aberration. Spherical aberration typically results in halos around point images and is reported to be one of the causes of night myopia. Also, the spherical aberration is commonly increased after myopic refractive surgery ${ }^{11}$. There is a relationship between the overall high order aberration and the pupil radius ${ }^{12}$, explained by the fact that most of the aberration is due to the second-order aberration, which has a square radius dependency.

In normal eyes, the ocular coma aberration increases with age mainly because of the increase in the corneal coma while the ocular spherical aberration increases with age due to the increase in spherical aberration in the internal optics, including the crystalline lens ${ }^{13}$. The coma aberration is often related with decentered corneal grafts, keratoconus, tilted suture-fixed intraocular lenses and decentered laser ablations. Also, it has been reported that high myopic eyes requiring larger amounts of laser correction and eyes undergoing conventional LASIK tend to induce more coma-like aberration after surgery ${ }^{14}$. The trefoil is reported to have a similar effect of coma on vision quality, although it produces less degradation in image quality compared with coma of similar root mean square (RMS) variance of the wave aberration wavefront.

The research performed in the area of ocular aberrations and its applications has contributed to the advance of ocular health, by providing the eye with a better retinal quality, and a better vision as a consequence, and improving the resolution in retinal imaging ${ }^{15,16}$.The precise determination and the consequent analysis of the optical aberrations are useful not only to understand the ocular system of the normal emmetropic eye, but perhaps the ability to measure accurately the optical quality of the ocular components is most powerful in the study of abnormal ocular optics. A combination of measurements between corneal topography and total aberrometry can result in a powerful tool for studies on pathologies of the human eye, changes induced by refractive surgery or design and in vivo testing of ophthalmic devices, such as intraocular or contact lenses.

In the field of teaching Vision Physics or Physiological Optics, the proper knowledge and analysis of the aberration that the human eye presents are of great interest, since this information allows a proper evaluation of the retinal image quality. The objective of the present work is to provide students the required competences needed to evaluate the optical quality of the human visual system for emmetropic and ammetropic eye, both with and without the optical compensation. For this purpose, an optical system corresponding to the Navarro-Escudero eye model, which allows calculating and evaluating the aberration of this eye model in different emmetropic and ammetropic conditions using OSLO LT software, was employed.

\section{MATERIALS AND METHODS}

\subsection{Navarro-Escudero schematic eye}

This eye model is based on a previously designed model with four centered aspheric surfaces to reproduce image quality on axis, and then transformed into a wide-angle model by simply adding a spherical image surface that plays the role of the retina ${ }^{17,18}$. This model captures the main features of the wide-angle optical design of the human eye with minimum complexity: four conic optical surfaces plus a spherical image surface. The modulation transfer function, Seidel 
aberrations (spherical aberration, coma, astigmatism, field curvature, and distortion), longitudinal and transverse chromatic aberrations, and overall monochromatic spot diagrams can be computed for this eye model and for field angles ranging from $0^{\circ}$ to $60^{\circ}$ by both finite and third-order ray tracing. This model is usually applied to simulate a variety of experimental methods in which image aberrations are estimated from measurements taken in the object space. Table 1 shows the parameter corresponding to the geometry of this schematic eye model

\begin{tabular}{|c|c|cc|}
\hline PARAMETER & Value & PARAMETER & Value \\
\hline Radius of curvature (mm) & \multicolumn{3}{c}{ Asphericity } \\
\hline Anterior surface of cornea & 7.72 & Cornea anterior & -0.26 \\
Posterior surface of cornea & 6.5 & Cornea posterior & 0 \\
Anterior surface of lens & 10.2 & Lens anterior & -3.1316 \\
Posterior surface of lens & -6 & Lens posterior & -1 \\
\hline Tickness (mm) & \multicolumn{3}{c}{ Refractive Index } \\
\hline Cornea & 0.55 & Cornea & 1.367 \\
Aqueous & 3.05 & Aqueous & 1.3374 \\
Lens & 4 & Lens & 1.42 \\
Vitreous & 16.4 & Vitreous & 1.336 \\
Resulting refractive power (diopters) 60.4 & \\
\hline
\end{tabular}

Table 1. Parameters of the Navarro-Escudero schematic eye model.

\section{2.- OSLO LT software}

For the development of the proposed practice course, we used the OSLO LT software, created by Lambda Research Corp, and which it can be downloaded through this link: http://www.sinopt.com/software1/downloads1/dloads_lt.htm

The OSLO LT software provides a computing environment for optical design. In addition to the usual functions that provide optimization and evaluation of optical systems, OSLO features a special windows interface that allows you to work interactively to probe the details of your optical system during the design process. OSLO accepts symbolic or numerical input using menus, toolbars, or commands; slider-wheel functions for real-time analysis; and automatic generation of dialog boxes and menus for custom program enhancements. OSLO works similarly to other Windows programs. If you are familiar with other Windows software, you will be able to use OSLO without difficulty. However, the OSLO user interface does contain several unique features that make it an efficient and easy-to-use optical design program.

Figure 1 shows a screen shot of a typical OSLO configuration. Generally, you enter data either in a spreadsheet or in the command line. You can enter commands either directly in the command line or by clicking a menu or toolbar button. Commands and menus are completely integrated; there are no separate command and graphical input modes. Output from OSLO typically appears in a text or graphics window, according to the type of output to be displayed. A unique feature of OSLO is its slider-wheel window, holding up to 32 graphical sliders, providing callbacks to default or user-supplied routines that perform evaluation or even full optimization iterations when a slider is moved. Other special windows are provided for database functions, file handling, and text editing. A substantial portion of OSLO is written in CCL, a modern byte-code language similar to Java. Incremental compilers and a linker are seamlessly integrated with the program to provide byte code efficiency with the ease of use of an interactive language.

The Standard Tools for graphic Windows are intended to provide general report graphic plots. More detailed analyses can be produced using subsidiary toolbars selected from the Window Setup menu (1 1 st toolbar button). The report 
graphics plots are made using CCL routines, so they can be modified to meet special requirements. The Standard Tools for graphics windows include basic lens drawing and optical evaluation features, such as:

- Ray Analysis report graphic window, which is a single plot that contains several ray-based evaluations: ray intercept curves, coddington astigmatism field curves, longitudinal spherical aberration, chromatic focal shift, distortion, and lateral color.. A plan view lens drawing is also shown in the lower right corner;

- Wavefront report graphic, which shows false-color interferograms and sliced-contour representations of the image-space wavefront for each field point;

- Spot diagrams for each field point. Although this is a vector plot, it is preferable to produce hard copy in the form of a bitmap to save file space;

- $\quad$ Point spread functions from the defined field points, together with $\mathrm{x}$ and y scans and encircled (and ensquared) energy distribution curves;

- $\quad$ MTF vs frequency and MTF vs focus plots. .

Although much optical design is done using graphics plots, some aspects require (numerical) text output. OSLO text windows have a unique feature for these aspects: the Spreadsheet buffer. The Spreadsheet buffer is an array in memory that mirrors text output sent to the display.

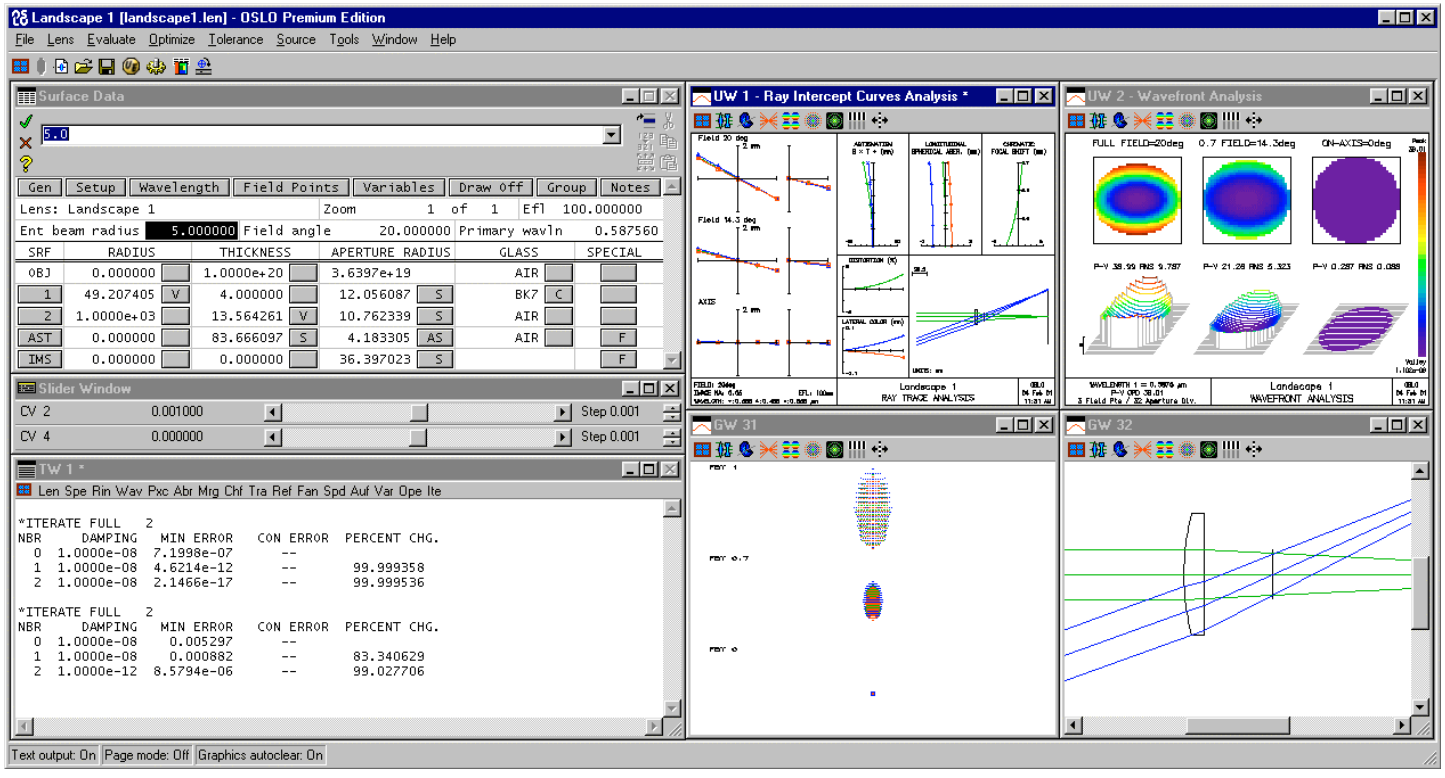

Figure 1. Screen shot of a typical configuration of the OSLO LT software.

\subsection{Design of the optical systems.}

5 lens files, designed according to the parameters of the Navarro-Escudero eye model (Table 1) but each corresponding to a different condition (emmetropic eye, myopic eye, hyperopic eye, compensated myopic eye and compensated hyperopic eye), were developed and are schematically represented in Figures 2-6. 


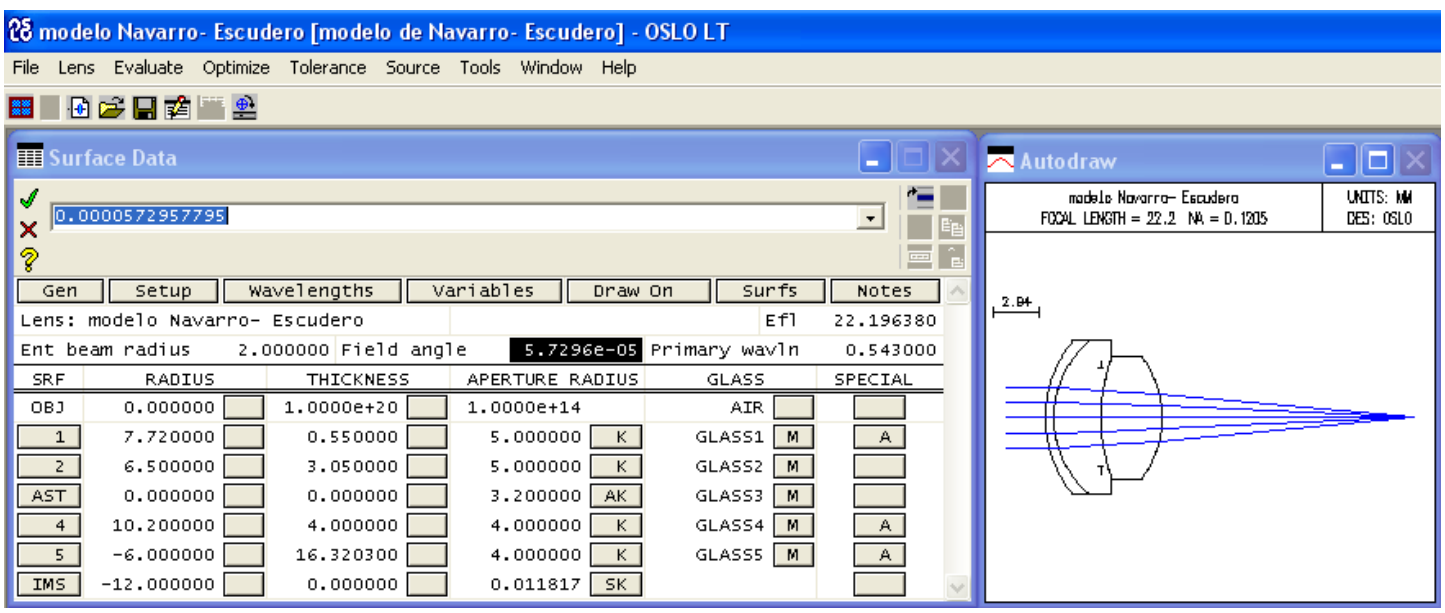

Figure 2. Lens file for emmetropic eye.

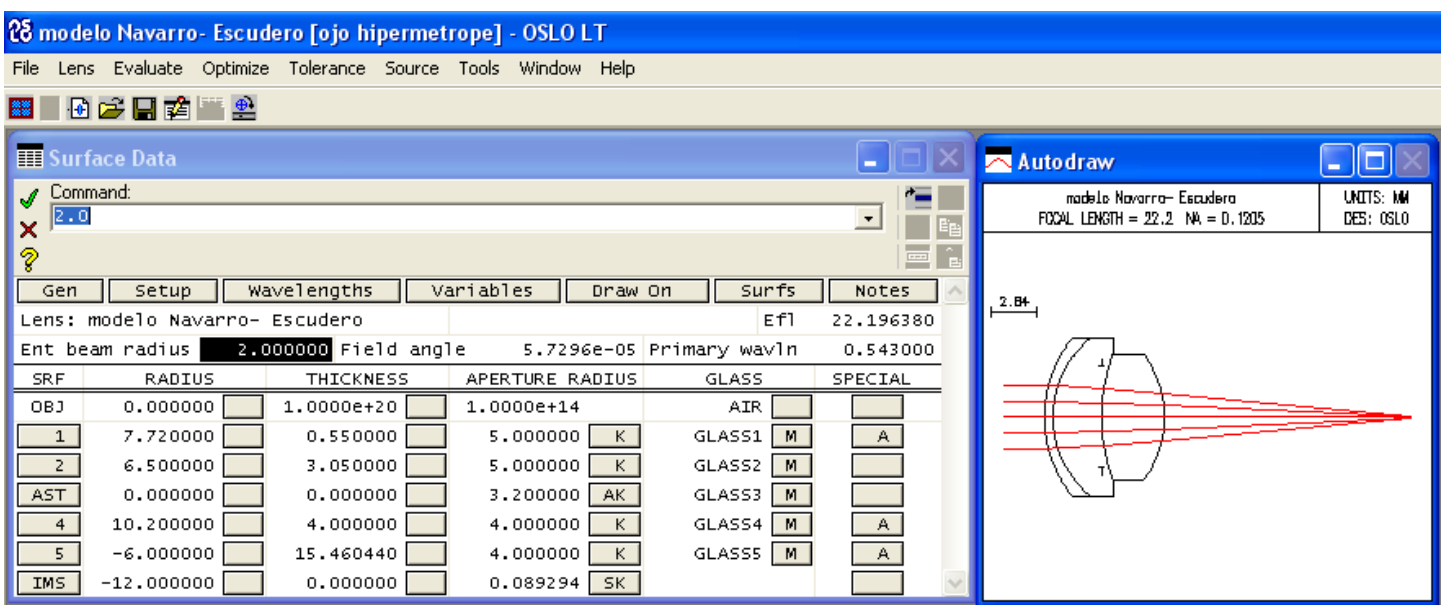

Figure 3. Lens file for hyperopic eye.

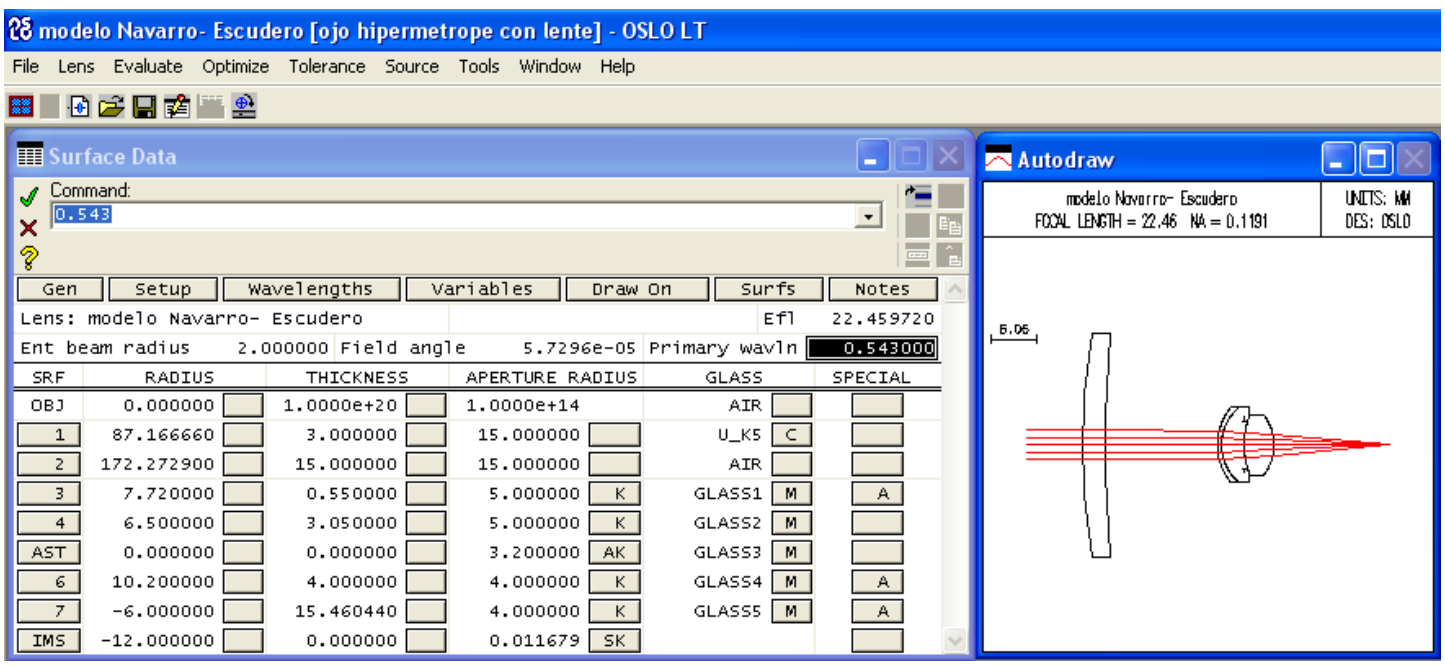

Figure 4. Lens file for compensated hyperopic eye. 


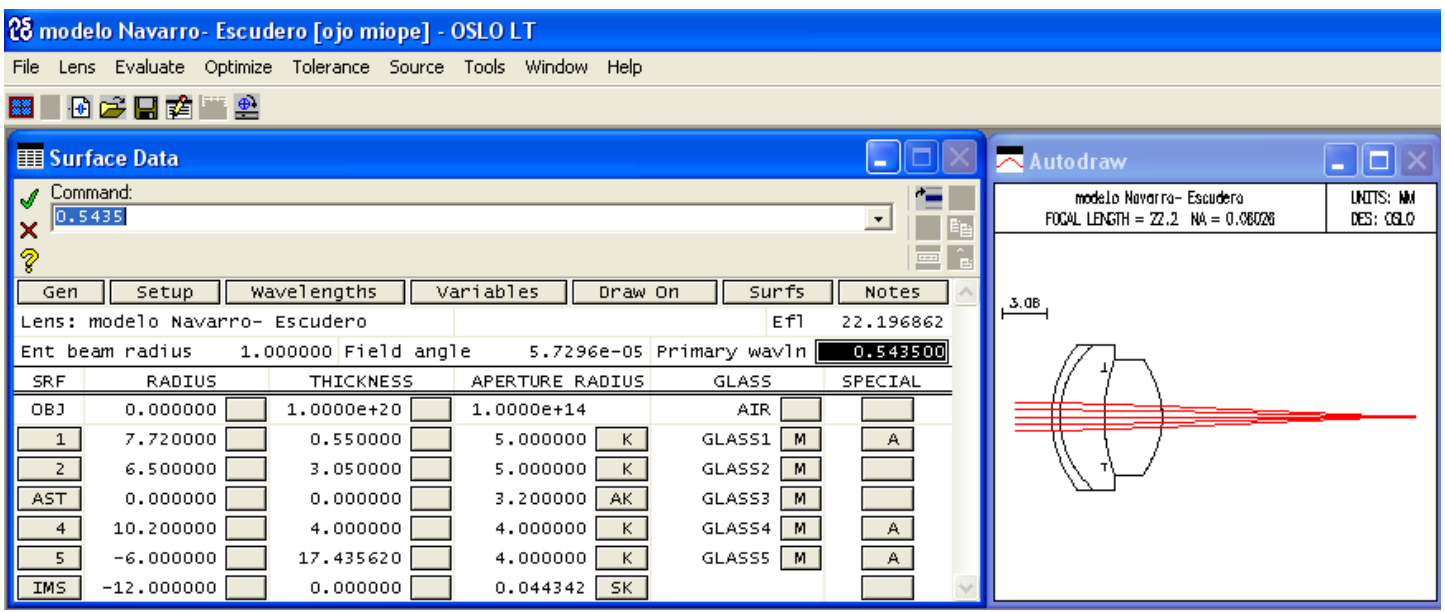

Figure 5. Lens file for myopic eye.

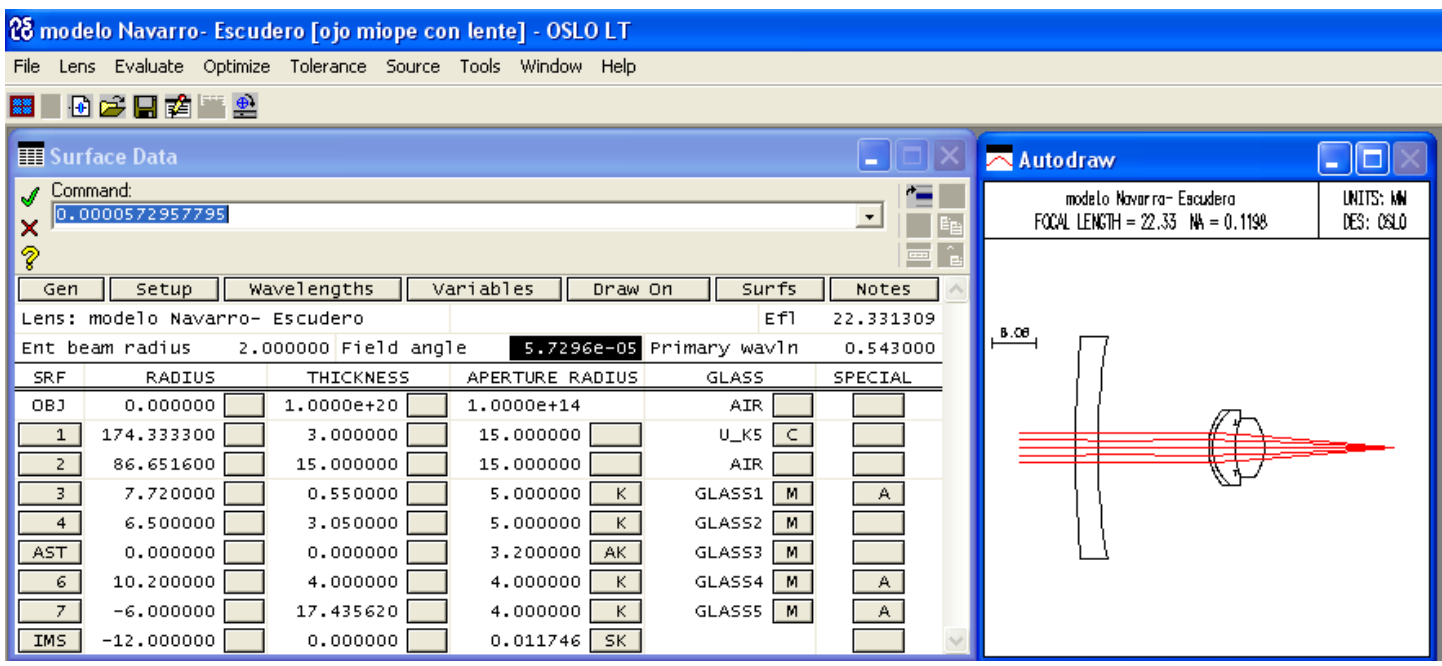

Figure 6. Lens file for compensated myopic eye.

We use as initial configuration for all lens files a beam radius of $2 \mathrm{~mm}$, field angle of $0^{\circ}$ and primary wavelength of 543nm. To reach our objective, the chromatic aberrations, Seidel aberrations and Fifth-order aberrations will be taken into account.

\section{STUDENT TASK: EVALUATION OF THE RETINAL IMAGE QUALITY}

The student evaluates the retinal image quality through determination and analysis of the Coddington astigmatism field curves, Longitudinal spherical aberration, chromatic focal shift, distortion, and lateral color, Spot diagrams for each field point, Point spread functions and MTF vs frequency and MTF vs focus plots for emmetropic eye, myopic eye, hyperopic eye, compensated myopic eye and compensated hyperopic eye. The student has to perform a comparison of the retinal image quality among the different types of eye models. The observation conditions were set as: beam radius $1-4 \mathrm{~mm}$ at $0.5 \mathrm{~mm}$ steps; field angle $0-60^{\circ}$ at $10^{\circ}$ steps and primary wavelength between $480-780 \mathrm{~nm}$ at $50 \mathrm{~nm}$ steps. 
3.1 Results of Coddington astigmatism field curves, Longitudinal spherical aberration, chromatic focal shift, distortion, and lateral color.

In Figures 7-9 are displayed some of the results obtained for Coddington astigmatism field curves, Longitudinal spherical aberration, chromatic focal shift, distortion, and lateral color for a beam radius of $3.5 \mathrm{~mm}$, field angle of $30^{\circ}$ and primary wavelength of 543nm.

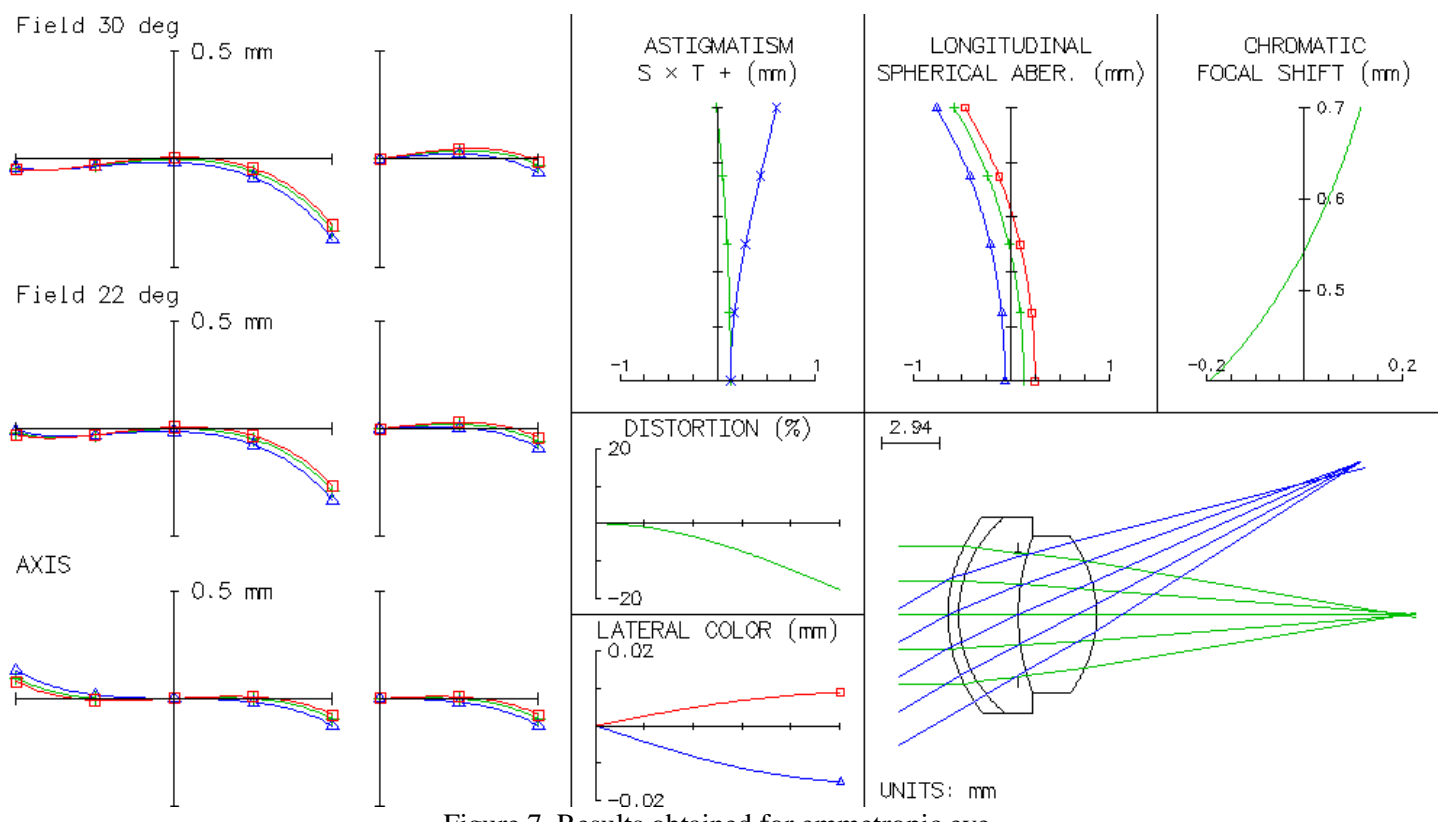

Figure 7. Results obtained for emmetropic eye.

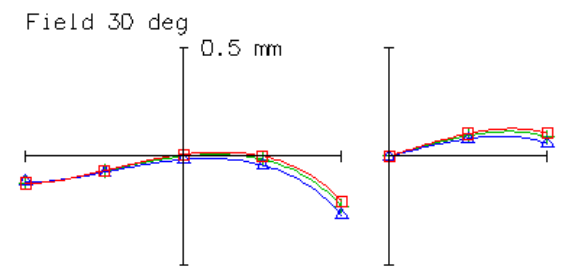

Field 22 deg

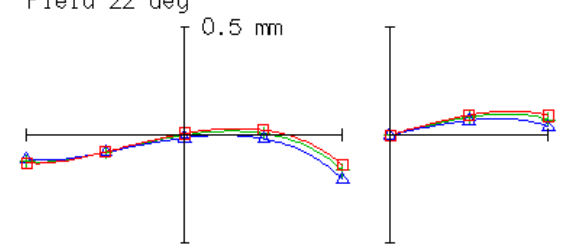

AXIS
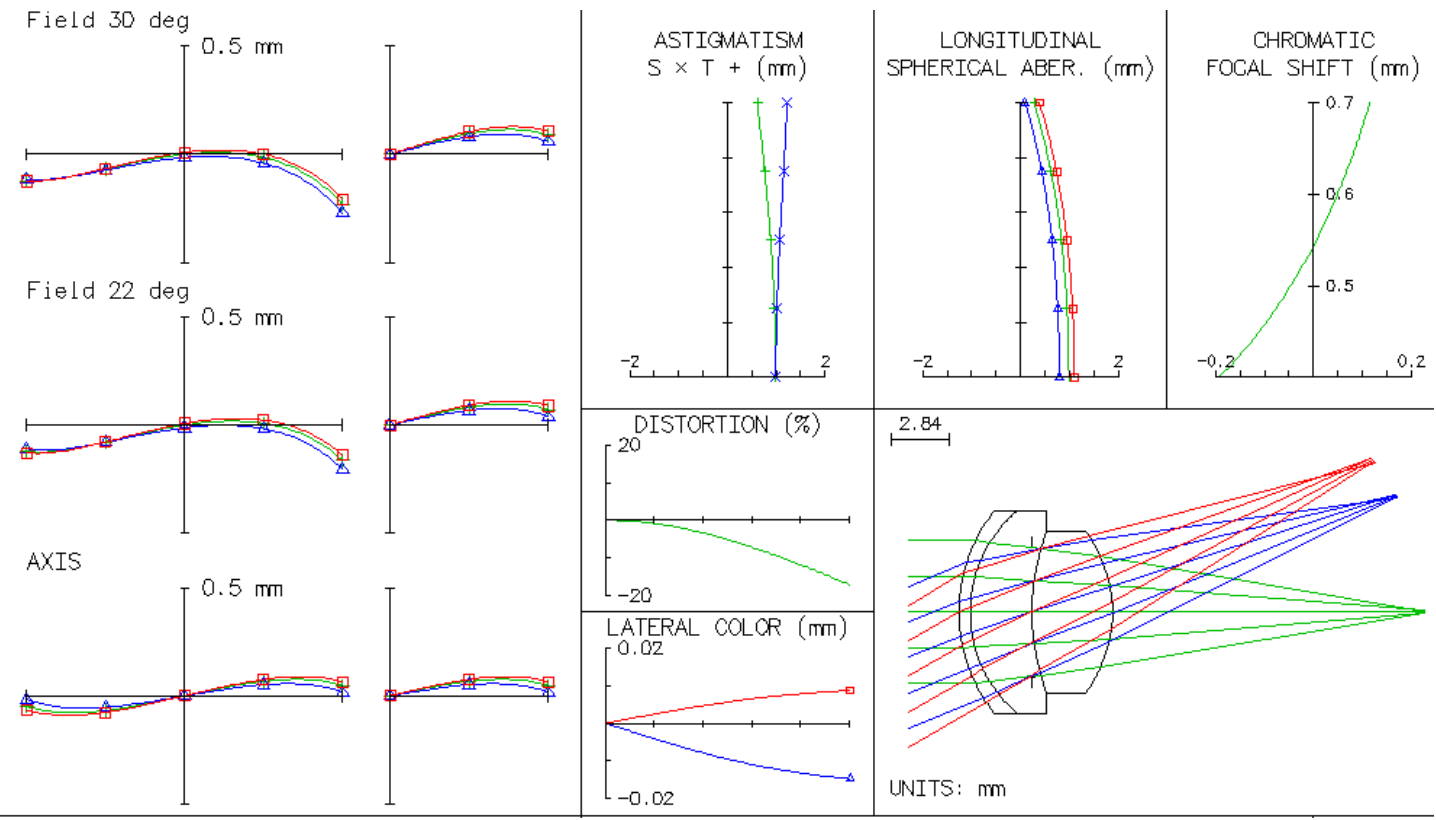

Figure 8. Results obtained for hyperopic eye. 


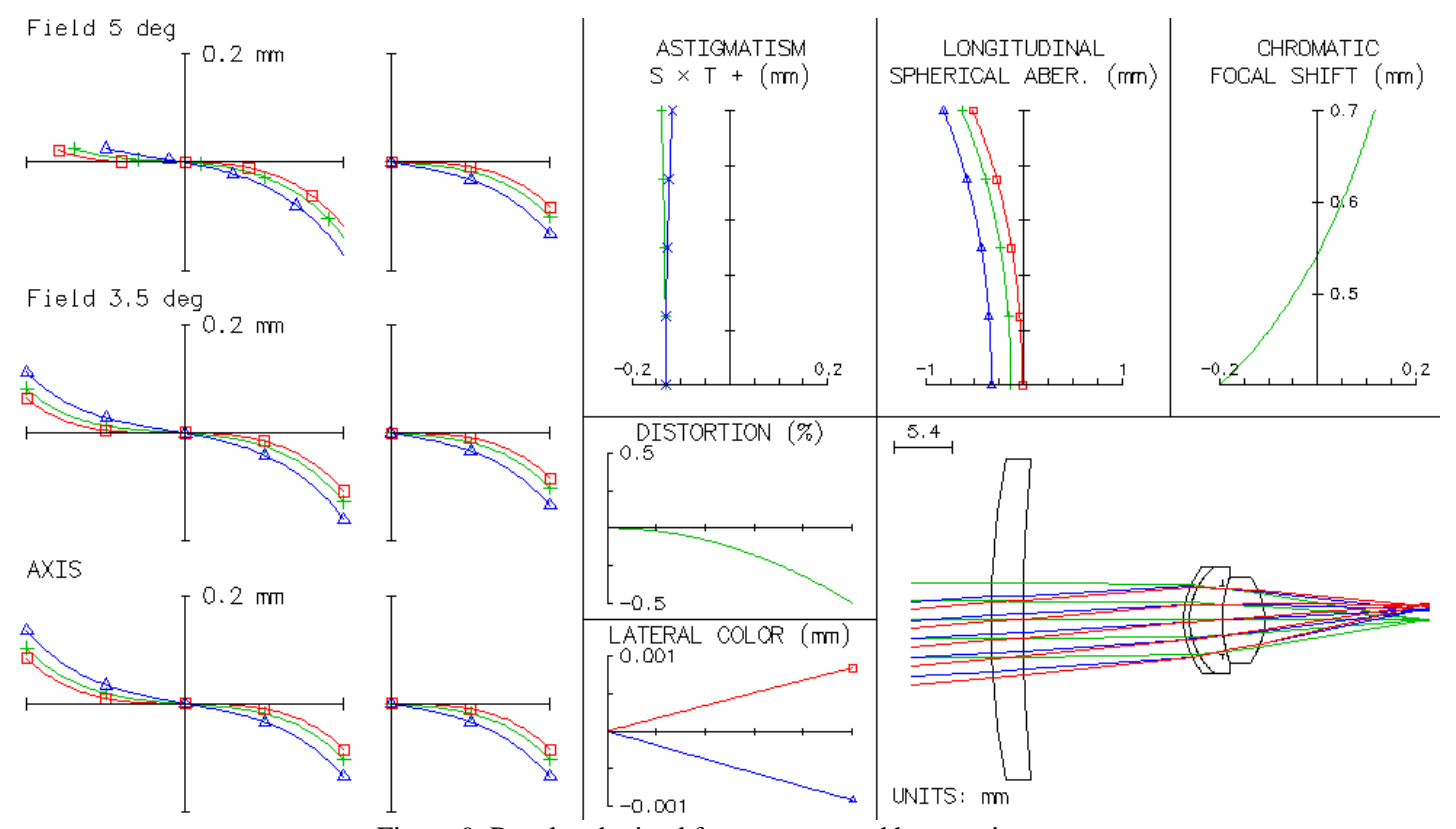

Figure 9. Results obtained for compensated hyperopic eye.

\subsection{Results of spot diagrams for each field point.}

In Figures 10 and 11 are displayed the spot diagrams for each field point obtained for a beam radius of $1 \mathrm{~mm}$, field angle of $30^{\circ}$ and primary wavelength of 543nm.

\section{FULL FIELD} 30deg

0.7 FIELD $22 \mathrm{deg}$

$\begin{array}{ll}\text { ON-AXIS } & \stackrel{\mid}{\circ} \\ \text { Odeg } & \vdots\end{array}$
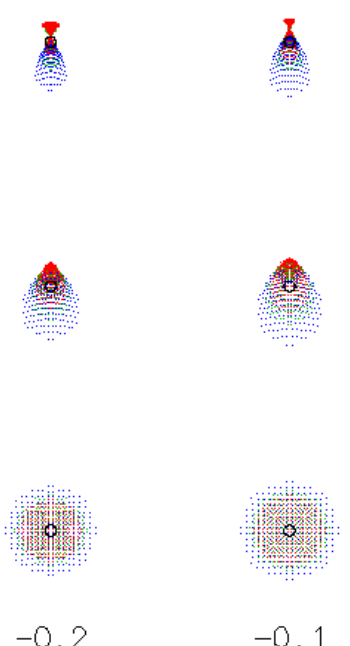

$-0.2$

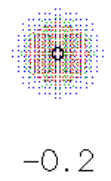

$-0.1$
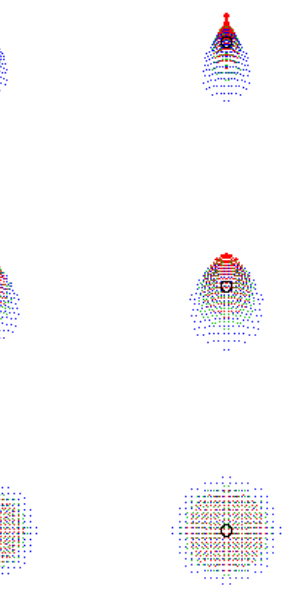

0
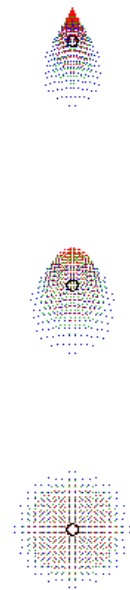

0.1
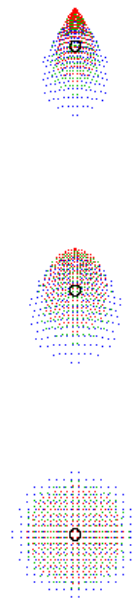

0.2

Figure 10. Spot diagrams for the myopic eye. 


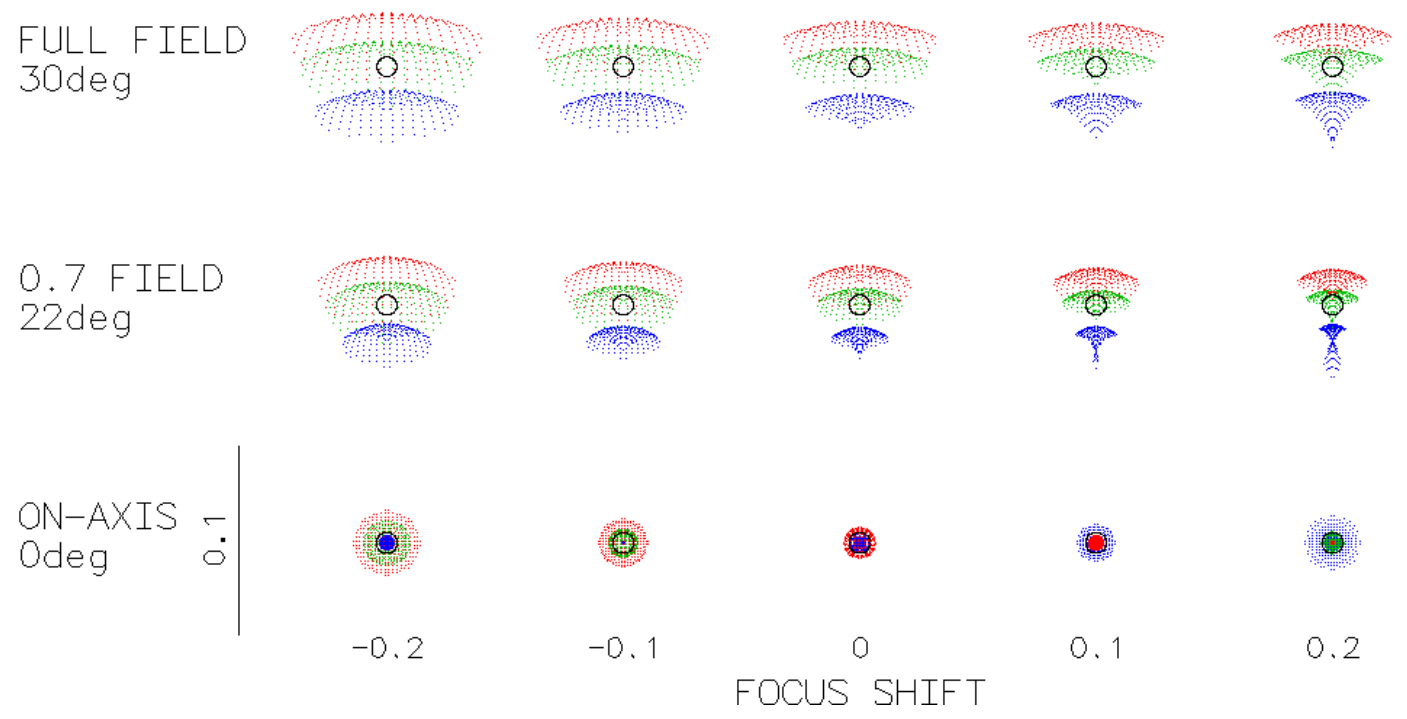

Figure 11. Spot diagrams for the compensated myopic eye.

\subsection{Modulation Transfer Function}

In Figures 12 and 13 are presented the Modulation Transfer Functions (MTF) obtained for a beam radius of $1 \mathrm{~mm}$, field angle of $30^{\circ}$ and primary wavelength of $543 \mathrm{~nm}$.

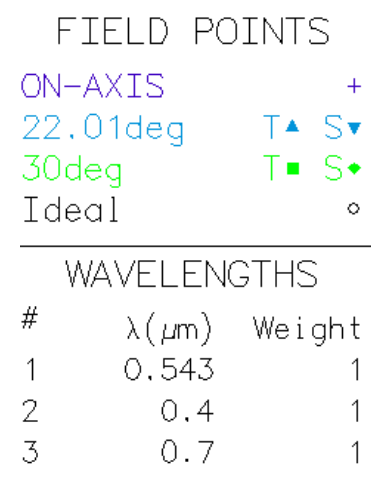

Proc. of SPIE Vol. 9289 92890R-9 


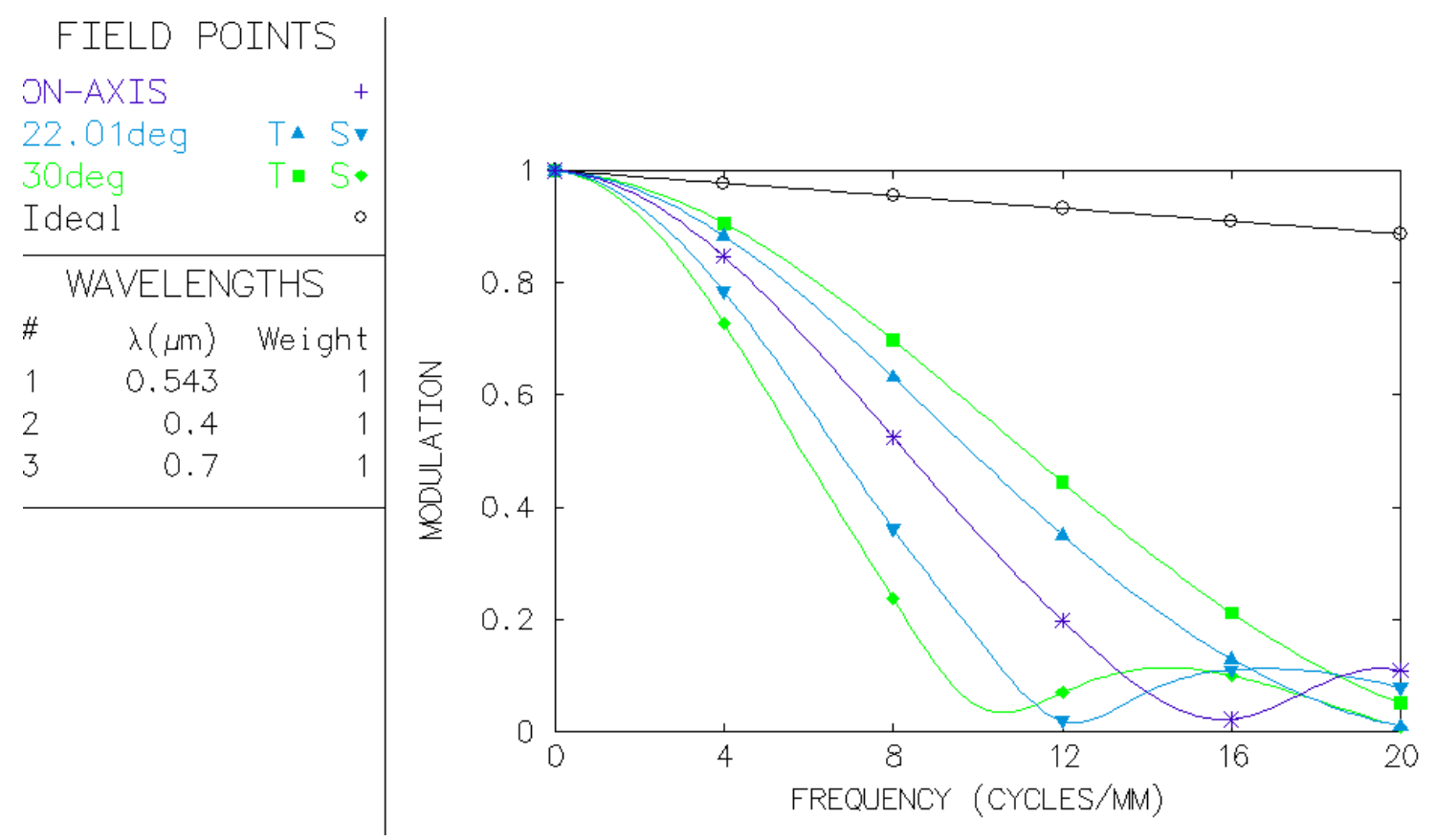

Figure 13. MTF of hyperopic eye.

\subsection{Seidel aberrations and Fifth-order aberrations}

In Table 2 are presented the the results obtained for the Seidel and Fifth-order aberrations for an emmetropic eye in the case of a beam radius of $1 \mathrm{~mm}, 2 \mathrm{~mm}$ and $3.5 \mathrm{~mm}, 0^{\circ}$ field angle, and primary wavelength of $543 \mathrm{~nm}$.

\begin{tabular}{|c|c|c|c|}
\hline & Emmetropic eye ( $\mathrm{r}=1 \mathrm{~mm})$ & Emmetropic eye (r=2mm) & Emmetropic eye $(r=3.5 \mathrm{~mm})$ \\
\hline \multicolumn{4}{|c|}{ Seidel Aberrations } \\
\hline SA3 & -0.0020 & -0.0168 & -0.0900 \\
\hline CMA3 & $-1.3 \cdot 10^{-8}$ & $-5.0 \cdot 10^{-8}$ & $-1.5 \cdot 10^{-7}$ \\
\hline AST3 & $-5.510^{-14}$ & $-1.110^{-13}$ & $-1.910^{-13}$ \\
\hline PTZ3 & $-3.610^{-13}$ & $-7.210^{-13}$ & $-1.210^{-12}$ \\
\hline DIS3 & $-2.110^{-18}$ & $-2.110^{-18}$ & $-2.110^{-18}$ \\
\hline \multicolumn{4}{|c|}{ Fifth-order Aberrations } \\
\hline SA5 & $-4.910^{-5}$ & -0.0016 & -0.0260 \\
\hline CMA5 & $1.110^{-10}$ & $1.810^{-9}$ & $1.710^{-8}$ \\
\hline AST5 & 0.0000 & 0.0000 & 0.0000 \\
\hline PTZ5 & 0.0000 & 0.0000 & 0.0000 \\
\hline DIS5 & 0.0000 & 0.0000 & 0.0000 \\
\hline
\end{tabular}

Table 2. Variations of the aberrations with different beam radius.

\section{CONCLUSIONS}

The course is expected to be of great interest for student of Optics and Optometry Sciences, last courses of Physics or medical sciences related with human vision, as a previous step of the clinical evaluation of patients. 


\section{REFERENCES}

[1] Atchinson, D.A., Smith, G., [Optics of the Human Eye], Butterworth-Heinemann, Oxford, United Kingdom, (2000).

[2] Welford, W.J., [Aberrations of optical systems], Adam Hilger Ltd, Bristol, United Kingdom, (1986).

[3] Malacara, D., [Optical Shop Testing $2^{\text {nd }}$ Ed], Wiley, New York, USA, (1992).

[4] Born, M., Wolf, E., [Principles of Optics $7^{\text {th }}$ Ed.], Cambridge University Press, United Kingdom, (1999).

[5] Zernike, von F., "Beugungstheorie des schneidenver-fahrens und seiner verbesserten form, der phasenkontrastmethode",.Physica I 1(8): 689-704 (1934).

[6] Thibos, L.N., Applegate, R.A., Schwiegerling, J.T., Webb, R., and VSIA Standards Taskforce MembersMembers, "Standars for reporting the optical aberrations of eyes," Journal of Refractive Surgery 18, S652-S660 (2002).

[7] Artal, P., Guirao, A., “Contributions of the cornea and the lens to the aberrations of the human eyes”, Opt Lett 23, 1713-1715 (1998).

[8] Smith, G., Cox, M.J., Calver, R., Garner, L.F., "The spherical aberration of the crystalline lens of the human eye”, Vision Res 41, 235-243 (2001).

[9] Plainis, S., Ginis, H.S., Pallikaris, A., "The effect of ocular aberrations on steady-state errors of accommodative response”, J Vis 5(5), 466-477, (2005).

[10] Tarrant, J., Roorda, A., Wildsoet, C.F., "Determining the accommodative response from wavefront aberrations", J Vis 10(5):4, 1-16, (2010).

[11] Queirós, A., Villa-Collar, C., González-Méijome, J.M., Jorge, J., Gutiérrez, A.R., “Effect of pupil size on corneal aberrations before and after standard laser in situ keratomileusis, custom laser in situ keratomileusis, and corneal refractive therapy”, Am J Ophthalmol 150(1), 97-109, (2010)

[12] Castejon-Mochon, J.F., Lopez-Gil, N., Benito, A., Artal, P., “Ocular wave-front aberration statistics in a normal young population”, Vision Research 42(13), 1611-1617 (2002).

[13] Oshika, T., “Quantitative assessment of quality of vision”, Nihon Ganka Gakkai Zasshi108(12), 770-807 (2004).

[14] Kamiya, K., Umeda, K., Igarashi, A., Ando, W., Shimizu, K., "Factors influencing the changes in coma-like aberrations after myopic laser in situ keratomileusis”, Curr Eye Res 36(10), 905-909 (2011).

[15] Burns, S.A., Marcos, S., Elsner, A.E., Bara, S., "Contrast improvement for confocal retinal imaging using phase correcting plates," Opt Lett 27, 400-402 (2002).

[16] Roorda, A., Romero-Borja, F., Donnelly, W.J., Queener, H., Hebert, T.J., Campbell, C.W., "Adaptive optics scanning laser ophthalmoscopy", Optics Express 10(9), 405-412 (2002).

[17] Navarro, R., Santamaría, J., Bescós, J., “Accommodation-dependent model of the human eye with aspherics”, JOSA A 2(8), 1273-1280 (1985).

[18] Escudero-Sanz, I., Navarro, R., “Off-axis aberrations of a wide-angle schematic eye model”, JOSA A 16(8), 18811891 (1999). 